\title{
A simple method to assess the oxidative susceptibility of low density lipoproteins \author{
and Ana María Cortizo*
} \\ Adriana E Scoccia, María Silvina Molinuevo, Antonio Desmond McCarthy
}

\author{
Address: Cátedra de Bioquímica Patológica, Facultad de Ciencias Exactas, Universidad Nacional de La Plata, La Plata, Argentina \\ E-mail: Adriana E Scoccia - ascoccia@biol.unlp.edu.ar; María Silvina Molinuevo - silmol@biol.unlp.edu.ar; Antonio \\ Desmond McCarthy - mccarthy@biol.unlp.edu.ar; Ana María Cortizo* - cortizo@biol.unlp.edu.ar \\ *Corresponding author
}

Published: 20 June 2001

BMC Clinical Pathology 200I, I:I

This article is available from: http://www.biomedcentral.com/I472-6890/I/I

(c) 200 I Scoccia et al, licensee BioMed Central Ltd.
Received: 9 March 200I

Accepted: 20 June 2001

\begin{abstract}
Background: Oxidative modification of low density lipoproteins (LDL) is recognized as one of the major processes involved in atherogenesis. The in vitro standardized measurement of LDL oxidative susceptibility could thus be of clinical significance. The aim of the present study was to establish a method which would allow the evaluation of oxidative susceptibility of LDL in the general clinical laboratory.

Results: LDL was isolated from human plasma by selective precipitation with amphipathic polymers. The ability of LDL to form peroxides was assessed by measuring thiobarbituric acid reactive substances (TBARS) after incubation with $\mathrm{Cu}^{2+}$ and $\mathrm{H}_{2} \mathrm{O}_{2}$. Reaction kinetics showed a three-phase pattern (latency, propagation and decomposition phases) which allowed us to select $150 \mathrm{~min}$ as the time point to stop the incubation by cooling and EDTA addition. The mixture $\mathrm{Cu}^{2+} /$ $\mathrm{H}_{2} \mathrm{O}_{2}$ yielded more lipoperoxides than each one on its own at the same time end-point. Induced peroxidation was measured in normal subjects and in type 2 diabetic patients. In the control group, results were $21.7 \pm 1.5 \mathrm{nmol} \mathrm{MDA} / \mathrm{mg} \mathrm{LDL}$ protein, while in the diabetic group results were significantly increased $(39.0 \pm 3.0 \mathrm{nmol} \mathrm{MDA} / \mathrm{mg} \mathrm{LDL}$ protein; $\mathrm{p}<0.00 \mathrm{I})$.
\end{abstract}

Conclusion: a simple and useful method is presented for the routine determination of LDL susceptibility to peroxidation in a clinical laboratory.

\section{Background}

Atherosclerosis is a pathology that affects many people and may cause their death or disability due to myocardial infarction or strokes. Although the clinical manifestations of the disease have been established, the underlying mechanism of atherogenesis is still unclear. Recent theory points toward the oxidative modification of LDL (LDL-Ox) as one of the major involved processes [1]. Nevertheless, hardly any of the biological effects of LDLOx have been tested in vivo.
Taking into account the potential clinical importance of the oxidative modification of LDL, many studies have been carried out to quantify their in vitro susceptibility to oxidation. This measurement is thought to correlate with the LDL oxidative susceptibility within the arterial wall [2].

Plasmatic LDLs may be isolated by different methods, which include sequential and density-gradient ultracentrifugation, chromatography, electrophoresis and selec- 
tive precipitation [3]. Lipid peroxidation is a very complex process that involves the chain reaction of free radicals with polyunsaturated fatty acids. These reactions lead to rearrangements of double bonds in conjugated dienes, hydroperoxide generation, lipid breakdown into lower molecular weight fragments, as well as chemical modifications in the apo B protein $[4,5,6,7,8]$. The extent of lipid peroxidation can be estimated by measurement of thiobarbituric reactive substances (TBARS). This method, although nonspecific, is of value in purified systems. TBARS determination mainly measures malondialdehyde (MDA) derived from the hydroperoxidation of unsaturated fatty acids with three or more double bonds.

Many studies have been carried out to establish the role of $\mathrm{Fe}^{3+}, \mathrm{Fe}^{2+}$ and $\mathrm{Cu}^{2+}$ in the oxidation of LDL $[1,8,9]$. In biological systems, the reduction of oxygen yields hydrogen peroxide and superoxide radical. The reaction between these two species generates a hydroxyl radical, which is the reactive oxygen species with the shortest half life and highest reactivity. This reaction, which is kinetically slow, can be accelerated by catalytic amounts of iron or copper salts [10].

In the present study we present a simple method which would allow the high-throughput routine evaluation of the oxidative susceptibility of LDLs in the simultaneous presence of $\mathrm{Cu}^{2+}$ and $\mathrm{H}_{2} \mathrm{O}_{2}$ in the general clinical laboratory. LDLs were isolated by selective precipitation and their oxidative susceptibility was evaluated through the quantitation of TBARS.

Table I: Recovery of I.44 nmol MDA/tube added to duplicated resuspended LDL samples obtained from three independent plasma samples.

\begin{tabular}{cccc}
\hline Sample & $\begin{array}{c}\text { nmol MDA / } \\
\text { tube in } \\
\text { the original } \\
\text { sample }\end{array}$ & $\begin{array}{c}\text { Observed } \\
\text { increment } \\
\text { (nmol MDA/ } \\
\text { tube) }\end{array}$ & mean \pm SD \\
A & 0.63 & 1.15 & $85 \pm 7$ \\
B & 0.69 & 1.30 & $78 \pm 12$ \\
C & 1.07 & 1.24 & $94 \pm 8$ \\
\hline & 0.42 & 1.00 & \\
\hline
\end{tabular}

\section{Results}

\section{Optimization of oxidative susceptibility assay}

As expressed in Materials and Methods, different volumes of solubilizing solution were used to resuspend LDL precipitates. Relatively low volumes $(0.4 \mathrm{ml})$ gave lower intra-assay coefficients of variation $(4.8 \%)$ than relatively high volumes of $1.0 \mathrm{ml}(\mathrm{CV}=10.8 \%)$. These results correspond to the analysis of 22 samples determined in duplicate. In order to assess the recovery of standard, a fixed amount of $1.44 \mathrm{nmol} \mathrm{MDA} \mathrm{/} \mathrm{tube} \mathrm{was}$ added to aliquots of previously assayed duplicated resuspended LDL samples from three different plasmas. As can be seen in Table 1 the MDA recovery varied between 78.1 and $93.8 \%$ of the true value.

In experiments aimed at adjusting the number of precipitate washes needed, LDL precipitate was washed once or twice with precipitating solution, or not washed at all, prior to solubilizing. The protein concentration and cholesterol content of the resulting resuspended LDL samples was then determined in duplicate. The first wash diminished the protein content of the resuspended LDL sample by $20 \%$, whereas the second wash further decreased protein content by $3 \%$. On the other hand, the cholesterol content of resuspended LDL samples did not vary as a consequence of successive washes ( $99 \pm 3$ and $98 \pm 2 \%$ of non washed precipitate, for 1 or 2 washes respectively). The samples were also submitted to agarose electrophoresis, and bands revealed with Coomasie brilliant blue, in order to evaluate the possible presence of contaminating plasma proteins. The unwashed precipitate showed a clearly visible band corresponding to albumin, as well as another band of greater intensity with the electrophoretic mobility of LDL. One and two washes with precipitating reagent greatly diminished - but did not completely eliminate - the albumin band, without provoking any changes in the intensity of the LDL band (data not shown). As the washing procedure eliminates non-apoB co-precipitating plasma proteins without cholesterol losses, a single wash was selected as the standard procedure.

Table 2: Effect of Triton X-I 00 on the LDL oxidative susceptibility assay.

\begin{tabular}{cc}
\hline Solubilizing reagent & $\begin{array}{c}\text { MDA nmol / } \mathrm{mg} \text { LDL protein } \\
\text { mean } \pm \text { SD }(\mathrm{n}=3)\end{array}$ \\
$\mathrm{NaCl}$ & $20.3 \pm 0.6$ \\
$\mathrm{NaCl}+$ Triton $\times-100$ & $21.0 \pm 0.7$
\end{tabular}

No significant differences between the two procedures were observed. 
In other experiments, the effect of the presence of Triton $\mathrm{X}-100$ in the solubilizing solution was evaluated by assaying the oxidative susceptibility in three independent precipitations of the same sample. Two solubilizing reagents were investigated, $50 \mathrm{~g} / \mathrm{l} \mathrm{NaCl}$ and $0.1 \%$ Triton $\mathrm{X}-$ 100 in $50 \mathrm{~g} / \mathrm{l} \mathrm{NaCl}$. Table 2 shows that the same results for LDL oxidative susceptibility were obtained with the two procedures. However, since resuspending the precipitate with Triton X-10o was found to be less time-consuming, it was chosen as the standard method.

The kinetics of $\mathrm{Cu}^{2+} / \mathrm{H}_{2} \mathrm{O}_{2}$-induced LDL peroxidation was monitored by measuring the TBARS levels in aliquots of three resuspended LDL samples incubated from 15 to 180 min with $\mathrm{Cu}^{2+} / \mathrm{H}_{2} \mathrm{O}_{2}$ (Figure 1). An initial lag phase could be observed with no increments in the absorbance, followed by another with a maximum slope (propagation phase). A final phase was evident with lower absorbance increments (decomposition phase). Figure 1 represents three examples of various LDL preparations. In most cases, the propagation phase reached a maximum at about $150 \mathrm{~min}$. Thus, this incubation period was selected for the LDL oxidation reaction. In further experiments, EDTA was validated as an effective inhibitor of the basal and induced oxidation reaction. After an incubation of $150 \mathrm{~min}$, the results for samples submitted to oxidation in the presence of EDTA (30 nmol/tube) monitored for TBARS formation, did not show significant differences when compared with the blanks (with $\mathrm{Cu}^{2+} / \mathrm{H}_{2} \mathrm{O}_{2}$, without sample). Thus, EDTA at this concentration was subsequently used to effectively stop the oxidative reaction induced by $\mathrm{Cu}^{2+} / \mathrm{H}_{2} \mathrm{O}_{2}$.

The basal LDL oxidation (as defined in Materials and Methods) was extremely low and was arbitrary assigned $100 \%$ value (Figure 2). In absence of EDTA and oxidating agents, the samples showed an inherent oxidability, with TBARS values of approximately twice that of basal LDL oxidation. We next analyzed the effect of $\mathrm{Cu}^{2+}$ and/ or $\mathrm{H}_{2} \mathrm{O}_{2}$ as inducers of LDL oxidation reaction. When added separately, $\mathrm{Cu}^{2+}$ and $\mathrm{H}_{2} \mathrm{O}_{2}$ increased TBARS formation by approximately 5 - fold. However; the simultaneous addition of $\mathrm{H}_{2} \mathrm{O}_{2}$ and $\mathrm{Cu}^{2+}$ induced a synergistic increase in TBARS levels (approximately 13- fold). Doubling doses of $\mathrm{Cu}^{2+}$ or $\mathrm{H}_{2} \mathrm{O}_{2}$ did not further increase the oxidation levels of LDL.

The influence of LDL protein content on the TBARS reaction, was evaluated by increasing the volume of resuspended LDL samples, under constant TBARS reagent volume and incubation period (Figure 3). The reaction was linear up to an LDL protein content of approximately $300 \mu \mathrm{g} /$ tube.

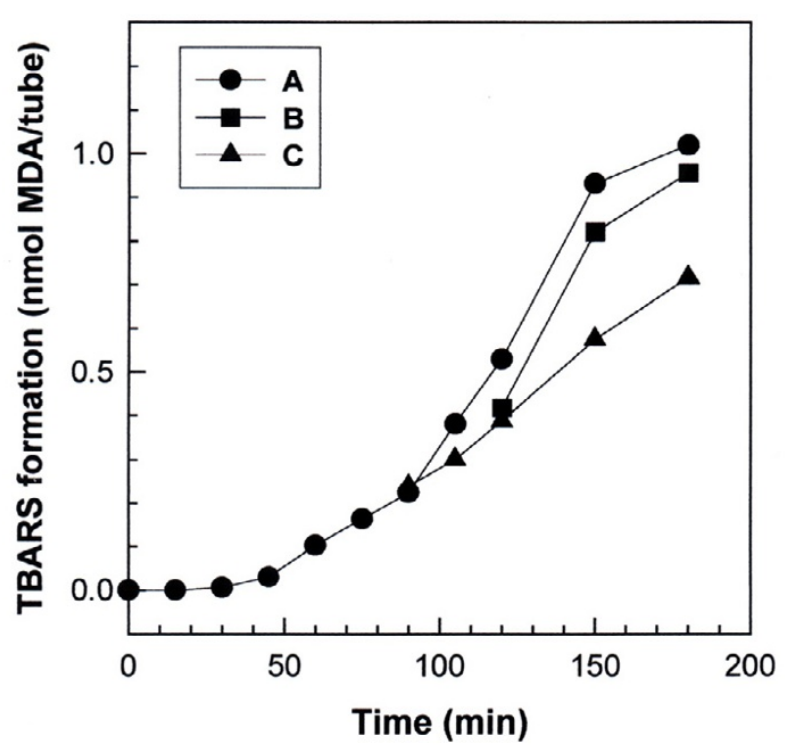

Figure I

Kinetics of in vitro LDL peroxidation assessed by TBARS formation. An aliquot of $100 \mu \mathrm{l}$ plasma from three different patients (A, B, C) was assayed. All sample precipitates were redissolved in $0.4 \mathrm{ml}$ of solubilizing solution, incubated with $\mathrm{Cu}^{2+} / \mathrm{H}_{2} \mathrm{O}_{2}$ for different periods of time, and 100 $\mu \mathrm{l}$ of oxidized LDL was employed for TBARS assay. Results are the average of duplicate determinations.

In order to characterize the LDL isolated by selective precipitation, and to investigate the possible damage of the inner structure of LDL caused by this method, we performed an agarose electrophoresis of the resuspended LDL sample in parallel with the LDL isolated by ultracentrifugation and the corresponding whole plasma sample. As can be seen in Figure 4, LDL fraction isolated by both methods showed the same electrophoretic mobility and no contamination by other lipoprotein fractions.

\section{LDL oxidation susceptibility in control and diabetic sam- ples}

In order to evaluate the method's usefulness in separating a control population from another with increased risk for cardiovascular disease, a group of 30 normal subjects and 12 type 2 diabetic patients were submitted to this assay. The oxidative susceptibility of LDL was significantly greater in the diabetic group than in the control population $(39.0 \pm 3.0 \mathrm{vs} .21 .7 \pm 1.5 \mathrm{nmol} \mathrm{MDA} / \mathrm{mg}$ LDL protein; $\mathrm{p}<0.001)$.

\section{Discussion}

The oxidative modification of LDL appears to be involved in the development of various degenerative dis- 


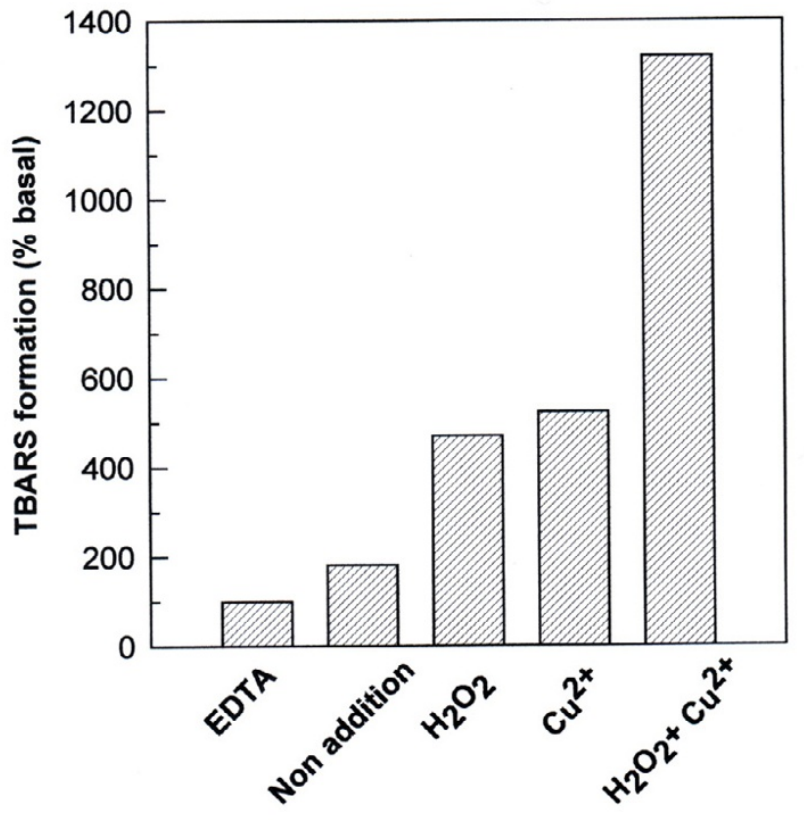

Figure 2

Basal and $\mathrm{Cu}^{2+}$ and $\mathrm{H}_{2} \mathrm{O}_{2}$-induced LDL oxidation. Samples were incubated with EDTA (basal LDL oxidation), without additions, or in the presence of $\mathrm{Cu}^{2+}(100 \mu \mathrm{M})$ and/ or $\mathrm{H}_{2} \mathrm{O}_{2}(300 \mathrm{ml} / \mathrm{l})$. Results are expressed as $\%$ basal TBARS values.

eases such as atherosclerosis, carcinogenesis, aging and diabetes mellitus $[11,12]$. Standard reference methods to prepare LDL from plasma employ ultracentrifugation. However, the selective precipitation methods, which are more accessible than ultracentrifugation, are widely used in the clinical laboratory for the measurement of the cholesterol content in different lipoprotein fractions. In particular, selective precipitation of LDL may be approached in different ways: by addition of heparin at an exactly controlled $\mathrm{pH}$ of 5.12 in the absence of divalent cations; or with polyvinylsulphate in the presence of EDTA and polyethylene glycol methyl ether; with amphipathic polymers in imidazole buffer at $\mathrm{pH}$ 6.10 (bioMerieux). An excellent statistical correlation is obtained when these methods are compared with reference ultracentrifugation methods, providing samples with triglyceride concentration above $8 \mathrm{mmol} / \mathrm{l}$ and those from patients with hyperlipoproteinemia Type III are excluded [13]. In particular, the precipitating reagent used in the present work (bioMerieux), shows a good correlation coefficient $(\mathrm{r}=0.96)$ when compared with ultracentrifugation methods [3]. Its selectivity and the preservation of the immunological properties as well as the lipid composition of the native original LDLs have also been demonstrated $[3,9,14,15]$. In our present study, we were unable to find differences in agarose electro- phoretic mobility between LDL fractions obtained by this method of selective precipitation and ultracentrifugation. In addition, no contaminating lipoprotein fractions were observed by this electrophoretic method. In our standard procedure we washed the LDL precipitate once prior to solubilizing. Thus, it was necessary to establish whether there were changes in LDL cholesterol content, which could invalidate the original method's correlation with ultracentrifugation. However, we were unable to find cholesterol losses as a consequence of one or two washes with precipitating reagent.

Arshad et al. [16] developed a simple method to assess whole plasma susceptibility to peroxidation by $\mathrm{Cu}^{2+} /$ $\mathrm{H}_{2} \mathrm{O}_{2}$ incubation. They used thiobarbituric acid reactivity to evaluate lipid peroxidation, a method which is not entirely specific. However, it proved to be easy to perform and accessible for the analysis of many samples. In the present work, we measured LDL-associated TBARS after induction of lipid peroxidation with a mixture of $\mathrm{Cu}^{2+}$ and $\mathrm{H}_{2} \mathrm{O}_{2}$.

Several methodological aspects of our procedure were subsequently addressed, in order to achieve its optimization. a) The intra-assay precision was found to depend on the volume of solubilizing solution employed. In our standard working conditions, the $\mathrm{CV}$ was $4.8 \%$, which is lower than the precision limit established for the determination of selectively precipitated lipoprotein cholesterol $(\mathrm{CV}<5 \%)$ [3], and so can be considered acceptable. b) The observed percentage of recovery for exogenously added MDA (Table 1) was comparable to that of the TBARS reaction (82-100 \%) [10]. These results suggest that the additioned MDA was still TBA reactive and did not generate any interfering substances, since the observed increment in MDA content did not significantly differ from that of the true value. c) It is important to ensure that the precipitate is not contaminated with nonLDL serum proteins, since results are expressed per LDL protein content. This contribution to variability was eliminated by washing the LDL precipitate. d) When the composition of the solubilizing solution was evaluated, precipitate redissolution effectively occurred in $50 \mathrm{~g} / \mathrm{l}$ $\mathrm{NaCl}$. However, the addition of Triton X-100 was chosen because it shortened the period of LDL redissolution. e) Lipid peroxidation kinetics have been extensively studied $[8,10]$. It is known that LDL oxidation in the presence of $\mathrm{Cu}^{2+}$ shows three phases: latency, propagation and decomposition. This has been established by determination of hydroperoxides, TBARS or other aldehydes, fluorescent products and conjugated dienes. It has been shown that during the latency and propagation phases, as well as during the early stages of the decomposition phase, the time-courses of diene, TBARS and lipid hydroperoxide formation, are practically coincident [8]. In- 
deed, the corresponding maxima coincide temporally. However, each individual's LDL shows its own particular kinetics so that sample to sample variations could represent a problem when - as in the present study - a single measurement of only one parameter is taken after a long incubation time. This does not allow us to conclusively establish whether the sample is at the end of its propagation phase, or has already begun its decomposition phase. In our preliminary studies of TBARS time-course, we found a lag phase followed by a maximum slope which ended at $150 \mathrm{~min}$, the time point adopted for our standard procedure. A slower increment in absorbance was observed from this point on, a fact that may have been due to the decomposition of accumulated products. f) In the absence of oxidation inhibitors, LDL oxidation may continue throughout the TBA reaction period, thus contributing to the method's variability. This was effectively prevented by the addition of EDTA prior to the TBA reaction, which acts as an inhibitor of LDL oxidation by $\mathrm{Cu}^{2+}$ sequestration. g) Our experiments show that the combination of $\mathrm{Cu}^{2+}$ and $\mathrm{H}_{2} \mathrm{O}_{2}$ is more effective for the induction of LDL oxidation, than each agent its own. The observed results suggest a synergistic mechanism of action between both reagents. Previous studies have addressed the $\mathrm{Cu}^{2+}$-induced in vitro oxidation of plasma LDL [17]. These authors found a value of $21 \pm 3$ nmol MDA / mg LDL protein, obtained from four normal subjects, for LDL isolated by ultracentrifugation. This is practically coincident with the results which we obtained with our control healthy population $(21.7 \pm 1.5 \mathrm{nmol}$ MDA / mg LDL protein), as would be expected from the reported correlation between LDL obtained by ultracentrifugation and by the LDL-precipitating method of bioMerieux. Recently, Guerci et al.[14] studied the LDL oxidation susceptibility of normolipidemic diabetic and non-diabetic patients. These authors found a significant increase in type 2 diabetic patients vs. healthy subjects, particularly in the group of type 2 diabetic females, in which LDL oxidation susceptibility was highest. In coincidence with these reported results, LDL oxidative susceptibility of our type 2 diabetic patients was significantly greater $(39.0 \pm 3.0 \mathrm{nmol} \mathrm{MDA} / \mathrm{mg} \mathrm{LDL}$ protein) than the control group.

The LDL precipitation method which we have used in this study is based on interaction with glycosaminoglycans (GAG). However, both lipid composition and the content of sialic acid can modulate the interaction with GAG. In this context, particles such as small dense LDL can interact with GAG with high affinity. In addition, the precipitation procedure may increase the susceptibility for oxidation by copper since copper penetrates the LDL particle more easily after precipitation. In consequence, we cannot discard the possibility that our results may reflect a preselection of LDL with higher susceptibility for oxidation.

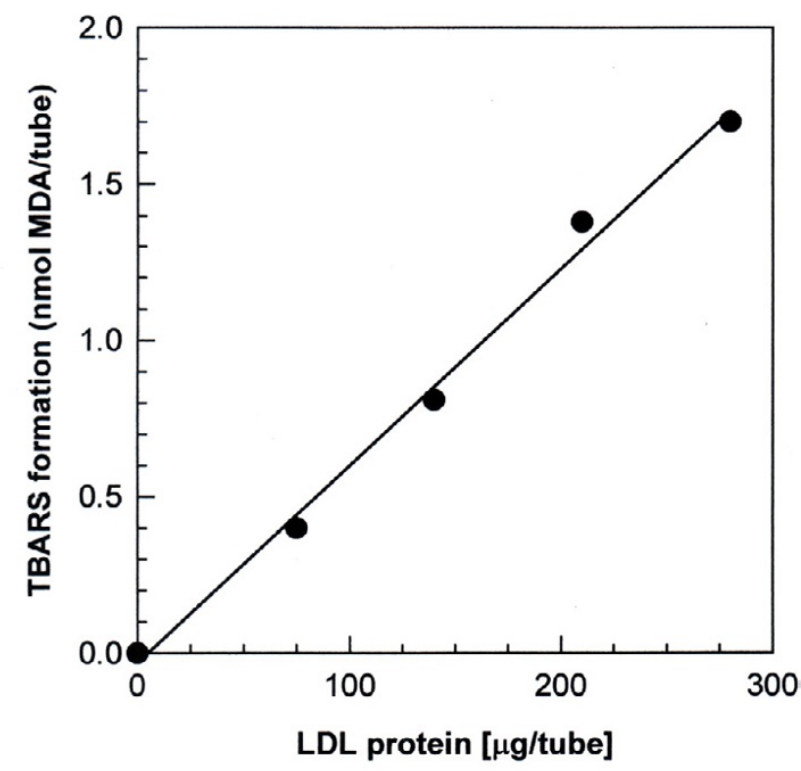

Figure 3

Influence of the LDL protein content on TBARS reaction linearity. Increasing doses of a LDL sample were submitted to constant oxidative conditions. Results are expressed as mean of duplicate determinations. $y=4.35 .10^{-}$ $3 \times-6.57 \cdot 10^{-3}, r^{2}=0.994 ; p<0.001$.

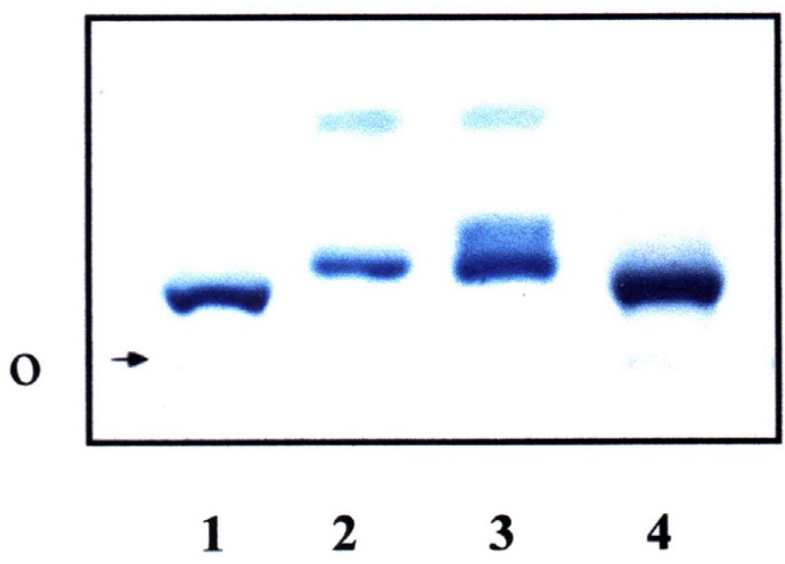

Figure 4

Agarose electrophoresis of whole plasma and LDL fractions. Bands correspond to: I, LDL fraction obtained by selective precipitation; 2 , whole plasma of a normolipidemic patient; 3 , whole plasma of a hyperlipidemic patient; 4 , LDL fraction obtained by ultracentrifugation. Samples were stained with Sudan black. 


\section{Conclusion}

A simple method for the in vitro measurement of LDL oxidation susceptibility has been optimized, and applied to a group of healthy subjects and type 2 diabetic patients. This straightforward approach could facilitate the comparison of results obtained from an increased number of general clinical laboratories, and thus allow us to move a step further towards the standardization of a procedure of potential clinical importance.

\section{Materials and methods \\ Materials}

LDL Cholesterol kit (cat. Number 61532) was provided by bioMerieux (Marcy l'Etoile France). Hydragel Lipo+Lp(a) kit for agarose electrophoresis was obtained from Sebia. Thiobarbituric acid was obtained from Merck. 1,1',3,3'- tetra-methoxy-propane or malondialdehyde (MDA) was used as standard and purchased from SIGMA Co. St. Louis, MO, USA. All chemicals were of analytical grade and used without further purification.

\section{Sample collection}

Twelve type 2 diabetic patients of both sexes (37- 65 years old) were studied. The degree of metabolic control was assessed by the measurement of fasting plasma glucose (mean $7.2 \pm 1.1 \mathrm{mmol} / \mathrm{l}$ ), fasting plasma $\mathrm{HbA}_{1 \mathrm{c}}$ (mean 6.8 $\pm 1.3 \%$; NV 4.8-6.0 \%) and they were normolipidemic. A series of 30 control non-diabetic subjects of both sexes (age range, 35-60 year old) was processed in parallel. All controls were normolipidemic according to the Alfedian criteria [18], and none of the subjects were taking any drug known to influence lipid or lipoprotein metabolism. Blood samples were obtained on heparin (5 $\mathrm{U} / \mathrm{ml}$ ) by venipuncture from subjects with 12 hours fasting. Plasma was separated rapidly and processed immediately. Alternatively, the samples were stored at $4{ }^{\circ} \mathrm{C}$ for 24 hours or at $-20^{\circ} \mathrm{C}$ for not more than 2 days.

\section{Method of LDL isolation}

LDL was selectively precipitated from $100 \mu \mathrm{l}$ of plasma by addition of bioMerieux precipitating reagent of LDLCholesterol kit and vortex-mixed [14]. The mixture was incubated for 30 min at $2-8{ }^{\circ} \mathrm{C}$, and centrifuged for 5 $\mathrm{min}$. The supernatant was discarded and the precipitate was washed with precipitating reagent. The washed precipitate was redissolved in different volumes of solubilizing solution (0.01\% Triton X100 in $50 \mathrm{~g} / \mathrm{l} \mathrm{NaCl}$ ) at $37^{\circ} \mathrm{C}$, and vortex-mixed (resuspended LDL sample) [15]. Bradford's method [19] was used to determine the total protein content of the resuspended LDL sample, using bovine serum albumin as a standard. For selected experiments, the LDL fraction was obtained by density gradient ultracentrifugation as has been previously described [13].

\section{Characterization of LDL isolated by selective precipitation} Representative samples were subjected to ultracentrifugation and selective precipitation (o, 1 or 2 washes) in order to isolate the LDL fraction. Subsequently, LDL fractions obtained by both methods, as well as the whole plasma, were electrophoresed in agarose according to the manufacturer's instructions. Briefly, electrophoresis was performed at a constant voltage of $130 \mathrm{~V}$ and initial intensity of $25 \mathrm{~mA}$, for 80 minutes. The gel was dried and bands were revealed with either Sudan Black or Coomasie brillant blue.

\section{LDL-cholesterol determination}

LDL was obtained by selective precipitation of representative samples, and the resulting precipitates were washed once, twice, or not at all with the precipitating reagent, prior to resuspending with the solubilizing solution. Cholesterol content of the resulting resuspended LDL samples was determined by a commercial enzymatic kit (Colestat, Wiener Laboratories Argentina).

\section{Basal and induced LDL oxidation}

Basal LDL oxidation was determined by incubating an aliquot of $100 \mu \mathrm{l}$ resuspended LDL sample, containing $50-90 \mu \mathrm{g}$ protein, with $30 \mu \mathrm{l}$ of $1 \mathrm{mM}$ EDTA and $45 \mu \mathrm{l}$ of distilled water. The corresponding blank was determined substituting the resuspended LDL sample by solubilizing solution.

In other experiments, resuspended LDL sample was mixed with $50 \mu \mathrm{l}$ of $100 \mu \mathrm{M} \mathrm{Cu}^{2+}$ (freshly prepared in phosphate buffer saline solution, $\mathrm{PBS}, \mathrm{pH}$ 7.4) and $25 \mu \mathrm{l}$ of $\mathrm{H}_{2} \mathrm{O}_{2}$ solution ( $300 \mathrm{ml} / \mathrm{l} \mathrm{H}_{2} \mathrm{O}_{2}$ in PBS, stock solution corresponds to 10 volume commercial $\mathrm{H}_{2} \mathrm{O}_{2}$ ). Blank was performed with solubilizing solution instead of resuspended LDL sample. In all cases, sample and blank were incubated at $37^{\circ} \mathrm{C}$ for different periods of time with occasional stirring. At the end of the incubation period, the lipid peroxidation was stopped by cooling and addition of $30 \mu \mathrm{l}$ of $1 \mathrm{mM}$ EDTA.

\section{TBARS determination}

Lipid peroxidation of LDL was assessed by TBARS formation [20]. Briefly, samples were incubated with $0.5 \mathrm{ml}$ of $20 \%$ acetic acid, $\mathrm{pH} 3.5$ and $0.5 \mathrm{ml}$ of $0.78 \%$ aqueous solution of thiobarbituric acid. After heating at $95{ }^{\circ} \mathrm{C}$ for 45 minutes, the samples were centrifuged at 4000 r.p.m. for 5 minutes. The red pigment in the supernatant fractions was estimated by absorbance at $532 \mathrm{~nm}$. A calibration curve was prepared with an MDA standard. Results were expressed as nmol MDA /mg LDL protein. All samples gave results which were within the linear portion of the MDA standard curve. A recovery assay was also performed by adding a defined amount of MDA before incubating with the oxidant mixture. 


\section{Statistical analysis}

Results were expressed as mean \pm SD and mean \pm SEM. Statistical analysis was performed by Student's t test; a p value $<0.05$ was considered statistically significant. Linear regression analysis was used for testing correlations between variables.

\section{Acknowledgements}

We thank Dra Susana Etcheverry for the kind revision of the paper. We are also grateful to $\mathrm{Dr} B$. Corsico for the preparation of LDL fraction by ultracentrifugation. AMC is a member of the Carrera del Investiga dor, CICPBA. This work was partially supported by grants from Facultad de Ciencias Exactas, UNLP, CICPBA. Authors thanks Dr Christian Coppens, Marcy, France and Dr Javier Goodman from bioMerieux Argentina for the provision of the LDL-cholesterol kit. We also thank Wiener Argentina for the donation of the Cholesterol kit.

\section{References}

I. Steinbergt D: Low density lipoprotein oxidation and its pathobiological significance. J Biol Chem 1997, 272:20963-20966

2. Schwartz CJ, Valente A: Atherogenesis and coronary heart disease: cellular mechanism. In International textbook of Diabetes Mellitus. Edited by Alberti KGMM, DeFronzo RA, Keen H, Zimmet $P$. Chichester: John Wiley, I997 I535-I54I

3. Rifai N, Warnick GR, McNamara JR, Belcher JD, Grinstead GF, Frantz ID Jr: Measurement of low-density- lipoprotein cholesterol in serum: a status report. Clin Chem 1992, 38:150-160

4. Esterbauer H, Jürgens G, Quehenberger O, Koller E: Autoxidation of human low density lipoprotein: loss of polyunsaturated fatty acids and vitamin $E$ and generation of aldehides. J Lipid Res 1987, 28:495-509

5. Buege JA, Aust SD: Microsomal lipid peroxidation. Methods Enzymol 1978, 52:302-310

6. Wallin B, Camejo G: Lipoprotein oxidation and measurement of hydroperoxide formation in a single microtitre plate. Scand J Clin Lab Invest 1994, 54:34I-346

7. El-Saadani M, Esterbauer H, El-Sayed M, Goher M, Nassar AY, Jürgens GA: Spectrophotometric assay for lipid peroxides in serum lipoproteins using a commercially available reagent. J Lipid Res 1989, 30:627-630

8. Esterbauer $\mathrm{H}$, Gebicki J, Puhl H, Jürgens $\mathrm{G}$ : The role of lipid peroxidation and antioxidants in oxidative modification of LDL. Free Radic Biol Med 1992, I 3:34 I-390

9. Taus M, Ferretti G, Dousset N, Moreau J, Battino M, Solera ML, Valdiguie $P$, Curatola $G$ : Suceptibility to in vitro lipid peroxidation of low density lipoproteins and erythrocyte membranes from liver cirrotic patients. Scand J Clin Lab Invest 1994, 54:147153

10. Coudray C, Richard MJ, Favier AE: Determination of primary and secondary lipid peroxidation products: plasma lipid hydroperoxides and thiobarbituric acid reactive substances. In Analysis of free radicals in biological systems. Edited by Favier AE, Cadet J, Kalyanaraman B, FontecaveM, Pierre JL. Base. Boston. Berlin: Birkhäuser Verlag, 1995185-200

II. Lyons TJ: Oxidized low density lipoproteins: a role in the pathogenesis of atherosclerosis in diabetes? Diabetes Med I991, 8:411-9

12. Steinberg D, Parthasarathy S, Carew TE, Khoo JC, Wilztum JL: Beyond cholesterol: modifications of low-density lipoprotein that increase its atherogenecity. $N$ Engl J Med I989, 320:915-24

13. Demacker PN, Hijmans AG, Brenninkmeijer BJ, Jansen AP, van't Laar A: Five methods for determining low -density lipoprotein cholesterol compared. Clin Chem 1994, 30:1797-1800

14. Guerci B, Antebi H, Meyer L, Durlach V, Ziegler O, Nicolas JP, Alcindor LG, Drouin P: Increased ability of LDL from normolipidemic Type 2 diabetic women to generate peroxides. Clin Chem 1999, 45:1439-1448

15. Moss MA, Wong CSY, Tan MH, Pett K, Jacklin CLE: Determination of low density lipoprotein cholesterol (LDL-C) in serum by BioMeriex cholesterol/phospholipids polyanions precipitation method and comparison with preparative ultracentrifugation. Clin Chem 1986, 32:1096-7
16. Arshad MAQ, Bhadra S, Cohen RM, Subbiah MTR: Plasma lipoprotein peroxidation potential: a test to evaluate individual susceptibility to peroxidation. Clin Chem 1991, 37:I756-I758

17. Lavy A, Brook GJ, Dankner G, Ben Amotz A, Aviram M: Enhanced in vitro oxidation of plasma lipoproteins derived from hypercholesterolemic patients. Metabolism 1991, 40:794-799

18. Brun JM, Droulin P, Berthezene F, Jacotot B, Pometta D: Dyslipidémies dy patient diabétique. Diabetes Metab 1995, 21:59-62

19. Bradford MM: A rapid and sensitive method for the quantitation of microgram quantities of protein utilizing the principle of protein-dye binding. Anal Biochem 1976, 72:243-254

20. Ohkawa $\mathrm{H}$, Ohishi N, Yagi K: Assay for lipid peroxides in animal tissues by thiobarbituric acid reaction. Anal Biochem 1979, 95:35l-358

\section{Pre-publication history}

The pre-publication history for this paper can be accessed here:

http://www.biomedcentral.com/content/backmatter/ 1472-6890-1-1-b1.pdf

Publish with BioMedcentral and every scientist can read your work free of charge

"BioMedcentral will be the most significant development for disseminating the results of biomedical research in our lifetime." Paul Nurse, Director-General, Imperial Cancer Research Fund

Publish with BMC and your research papers will be:

- available free of charge to the entire biomedical community

- peer reviewed and published immediately upon acceptance

- cited in PubMed and archived on PubMed Central

- yours - you keep the copyright 